\title{
SOJOURN TIMES
}

\author{
LAJOS TAKÁCS ${ }^{1}$ \\ Case Western Reserve University, Department of Mathematics \\ Cleveland, OH 44106 USA
}

(Received April, 1996; Revised July, 1996)

\begin{abstract}
Let $\{\zeta(u), u \geq 0\}$ be a stochastic process with state space $A \cup B$ where $A$ and $B$ are disjoint sets. Denote by $\beta(t)$ the total time spent in state $B$ in the interval $(0, t)$. This paper deals with the problem of finding the distribution of $\beta(t)$ and the asymptotic distribution of $\beta(t)$ as $t \rightarrow \infty$ for various types of stochastic processes. The main result is a combinatorial theorem which makes it possible to find in an elementary way, the distribution of $\beta(t)$ for homogeneous stochastic processes with independent increments.
\end{abstract}

This article is dedicated to the memory of Roland L. Dobrushin.

Key words: Stochastic Processes, Sojourn Times, Distributions, Limit Distributions

AMS (MOS) subject classifications: $60 \mathrm{G} 50,60 \mathrm{~J} 55$.

\section{Introduction}

Let $\{\zeta(u), u \geq 0\}$ be a stochastic process with state space $A \cup B$ where $A$ and $B$ are disjoint sets. If $\zeta(u) \in A$, then we say that the process is in state $A$ at time $u$, and if $\zeta(u) \in B$, then we say that the process is in state $B$ at time $u$. Denote by $\alpha(t)$ the total time spent in state $A$ in the time interval $(0, t)$ and by $\beta(t)$ the total time spent in state $B$ in the time interval $(0, t)$. Clearly, $\alpha(t)+\beta(t)=t$ for all $t \geq 0$. Our aim is to find the distribution of $\beta(t)$ and the asymptotic distribution of $\beta(t)$ as $t \rightarrow \infty$ for various types of stochastic processes.

Sojourn time problems have been studied extensively in the theory of probability. In 1939, P. Lévy $[13,14]$ obtained some basic results for the sojourn time of Brownian motion. Let $\{\xi(u), u \geq 0\}$ be a standard Brownian motion process. We have $\mathbf{P}\{\xi(u) \leq x\}=\Phi(x / \sqrt{u})$ for $u>0$ where

$$
\Phi(x)=\frac{1}{\sqrt{2 \pi}} \int_{-\infty}^{x} e^{-u^{2} / 2} d u
$$

is the normal distribution function. We use the notation $\delta(S)$ for the indicator variable of an event $S$, that is, $\delta(S)=1$ if $S$ occurs, and $\delta(S)=0$ if $S$ does not occur. Define

$$
\tau(\alpha)=\int_{0}^{1} \delta(\xi(u) \leq \alpha) d u,
$$

that is, $\tau(\alpha)$ is the sojourn time of the process $\{\xi(u), u \geq 0\}$ spent in the set $(-\infty, \alpha]$ in the time

${ }^{1}$ Postal address: 2410 Newbury Drive, Cleveland Heights, Ohio 44118, USA. 
interval $(0,1)$. If $\alpha \geq 0$, then

$$
\mathbf{P}\{\tau(\alpha) \leq x\}=\frac{1}{\pi} \int_{0}^{x} \frac{e^{-\alpha^{2} /(2 u)}}{\sqrt{u(1-u)}} d u
$$

for $0 \leq x<1$, and

If $\alpha=0$, then

$$
\mathbf{P}\{\tau(\alpha)=1\}=2 \Phi(\alpha)-1
$$

$$
\mathbf{P}\{\tau(0) \leq x\}=\frac{2}{\pi} \arcsin \sqrt{x}
$$

for $0 \leq x \leq 1$. Formula (5) was found by P. Lévy [13, 14, p. 303] in 1939, and is called the arcsine law. The more general result (3) was also found by P. Lévy [14, p. 326] but in a form more complicated than (3). The above form is given by M. Yor [24].

In 1949, M. Kac [11] gave a general method of finding the distribution of the random variable

$$
\sigma(t)=\int_{0}^{t} V(\xi(u)) d u
$$

for the Brownian motion $\{\xi(u), u \geq 0\}$ where $V(x)$ is a given function subject to certain restrictions. If, in particular, $V(x)$ is the indicator function of the set $(-\infty, \alpha]$ and $t=1$, then $\sigma(t)$ reduces to $\tau(\alpha)$ defined by (2). M. Kac [11] showed that the double Laplace transform of $\sigma(t)$ can be obtained by solving the differential equation

$$
\frac{1}{2} \frac{\partial^{2} \Psi}{\partial x^{2}}-(s+u V(x)) \Psi=0, \quad x \neq 0,
$$

subject to the conditions $\Psi(x) \rightarrow 0$ as $x \rightarrow \pm \infty,\left|\Psi^{\prime}(x)\right|<M$ for $x \neq 0, \Psi^{\prime}(+0)-\Psi^{\prime}(-0)=-2$. In 1957, D.A. Darling and M. Kac [5] considered the problem of finding the asymptotic distribution of $\sigma(t)$ for a Markov process $\{\xi(u), u \geq 0\}$.

\section{A Combinatorial Theorem}

For any $n$ real numbers $x_{1}, x_{2}, \ldots, x_{n}$ define $f_{n, k}\left(x_{1}, x_{2}, \ldots, x_{n}\right), 0 \leq k \leq n$, as the elements of the sequence

$$
\left\{0, x_{1}, x_{1}+x_{2}, \ldots, x_{1}+x_{2}+\ldots+x_{n}\right\}
$$

arranged in nondecreasing order of magnitude, that is,

$$
f_{n, 0}\left(x_{1}, x_{2}, \ldots, x_{n}\right) \leq f_{n, 1}\left(x_{1}, x_{2}, \ldots, x_{n}\right) \leq \ldots \leq f_{n, n}\left(x_{1}, x_{2}, \ldots, x_{n}\right) .
$$

In particular,

and

$$
f_{n, 0}\left(x_{1}, x_{2}, \ldots, x_{n}\right)=\min \left(0, x_{1}, x_{1}+x_{2}, \ldots, x_{1}+x_{2}+\ldots+x_{n}\right)
$$

$$
f_{n, n}\left(x_{1}, x_{2}, \ldots, x_{n}\right)=\max \left(0, x_{1}, x_{1}+x_{2}, \ldots, x_{1}+x_{2}+\ldots+x_{n}\right) .
$$

Furthermore, define $g_{n, k}\left(x_{1}, x_{2}, \ldots, x_{n}\right)$ for $0 \leq k \leq n$ as follows:

and

$$
\begin{aligned}
& g_{n, 0}\left(x_{1}, x_{2}, \ldots, x_{n}\right)=f_{n, 0}\left(x_{1}, x_{2}, \ldots, x_{n}\right), \\
& g_{n, n}\left(x_{1}, x_{2}, \ldots, x_{n}\right)=f_{n, n}\left(x_{1}, x_{2}, \ldots, x_{n}\right),
\end{aligned}
$$

for $1 \leq k<n$.

$$
\begin{gathered}
g_{n, k}\left(x_{1}, x_{2}, \ldots, x_{n}\right)=\max \left(0, x_{1}, x_{1}+x_{2}, \ldots, x_{1}+x_{2}+\ldots+x_{k}\right) \\
+\min \left(0, x_{k+1}, x_{k+1}+x_{k+2}, \ldots, x_{k+1}+\ldots+x_{n}\right)
\end{gathered}
$$

Let $c_{1}, c_{2}, \ldots, c_{n}, \ldots$ be a sequence of real numbers and denote by $C_{n}$ the set of all $n$ ! permuta- 
tions of $\left(c_{1}, c_{2}, \ldots, c_{n}\right)$. Define the following sets:

and

$$
\boldsymbol{F}_{n, k}\left(c_{1}, c_{2}, \ldots, c_{n}\right)=\left\{f_{n, k}\left(x_{1}, x_{2}, \ldots, x_{n}\right):\left(x_{1}, x_{2}, \ldots, x_{n}\right) \in \boldsymbol{C}_{n}\right\}
$$

for $0 \leq k \leq n$.

$$
G_{n, k}\left(c_{1}, c_{2}, \ldots, c_{n}\right)=\left\{g_{n, k}\left(x_{1}, x_{2}, \ldots, x_{n}\right):\left(x_{1}, x_{2}, \ldots, x_{n}\right) \in C_{n}\right\}
$$

We have

$$
\boldsymbol{G}_{1,0}\left(c_{1}\right)=\boldsymbol{F}_{1,0}\left(c_{1}\right)
$$

because $g_{1,0}\left(c_{1}\right)=f_{1,0}\left(c_{1}\right)=\min \left(0, c_{1}\right)$ and

$$
\boldsymbol{G}_{1,1}\left(c_{1}\right)=\boldsymbol{F}_{1,1}\left(c_{1}\right)
$$

because $g_{1,1}\left(c_{1}\right)=f_{1,1}\left(c_{1}\right)=\max \left(0, c_{1}\right)$.

Theorem 1: For every $n=1,2, \ldots$ and $k=0,1, \ldots, n$ and for every choice of the sequence $c_{1}, c_{2}, \ldots, c_{n}, \ldots$, the two sets $\boldsymbol{F}_{n, k}\left(c_{1}, c_{2}, \ldots, c_{n}\right)$ and $\boldsymbol{G}_{n, k}\left(c_{1}, c_{2}, \ldots, c_{n}\right)$ contain exactly the same elements.

Proof: We shall prove the theorem by mathematical induction. If $n=1$, then by (17) and (18) the statement is true for $k=0$ and $k=1$. Let us assume that the statement is true for a positive integer $n$ and every $k=0,1, \ldots, n$. We shall prove that it is true for $n+1$ and $k=0,1$, $\ldots, n+1$. This implies that the statement is true of all $n=1,2, \ldots$ and $0 \leq k \leq n$. The proof is based on the following recurrence formulas:

$$
f_{n+1, k}\left(c_{1}, c_{2}, \ldots, c_{n+1}\right)=\left[c_{1}+f_{n, k-1}\left(c_{2}, \ldots, c_{n+1}\right)\right]^{+}+\left[c_{1}+f_{n, k}\left(c_{2}, \ldots, c_{n+1}\right)\right]^{-}
$$

and

$$
\begin{gathered}
g_{n+1, k}\left(c_{1}, c_{2}, \ldots, c_{n+1}\right)=\left[c_{1}+g_{n, k-1}\left(c_{2}, \ldots, c_{n+1}\right)\right]^{+} \\
+\left[c_{k+1}+g_{n, k}\left(c_{1}, \ldots, c_{k}, c_{k+2}, \ldots, c_{n+1}\right)\right]^{-}
\end{gathered}
$$

for $1 \leq k \leq n$ where $[x]^{+}=\max (0, x)$ and $[x]^{-}=\min (0, x)$. Formulas (19) and (20) follow immediately from the definitions of $f_{n, k}$ and $g_{n, k}$.

We have

$$
f_{n+1, k}\left(c_{1}, \ldots, c_{n+1}\right)>0 \text { if and only if } c_{1}+f_{n, k-1}\left(c_{2}, \ldots, c_{n+1}\right)>0
$$

and then

Also,

$$
f_{n+1, k}\left(c_{1}, \ldots, c_{n+1}\right)=c_{1}+f_{n, k-1}\left(c_{2}, \ldots, c_{n+1}\right) .
$$

and then

$$
f_{n+1, k}\left(c_{1}, \ldots, c_{n+1}\right)<0 \text { if and only if } c_{1}+f_{n, k}\left(c_{2}, \ldots, c_{n+1}\right)<0
$$

$$
f_{n+1, k}\left(c_{1}, \ldots, c_{n+1}\right)=c_{1}+f_{n, k}\left(c_{2}, \ldots, c_{n+1}\right) .
$$

In any other case $f_{n+1, k}\left(c_{1}, \ldots, c_{n+1}\right)=0$.

Furthermore, we have

$$
g_{n+1, k}\left(c_{1}, \ldots, c_{n+1}\right)>0 \text { if and only if } c_{1}+g_{n, k-1}\left(c_{2}, \ldots, c_{n+1}\right)>0
$$

and then

Also,

$$
g_{n+1, k}\left(c_{1}, \ldots, c_{n+1}\right)=c_{1}+g_{n, k-1}\left(c_{2}, \ldots, c_{n+1}\right) .
$$

$$
g_{n+1, k}\left(c_{1}, \ldots, c_{n+1}\right)<0 \text { if and only if } c_{k+1}+g_{n, k}\left(c_{1}, \ldots, c_{k}, c_{k+2}, \ldots, c_{n+1}\right)<0
$$

and then

$$
g_{n+1, k}\left(c_{1}, \ldots, c_{n+1}\right)=c_{k+1}+g_{n, k}\left(c_{1}, \ldots, c_{k}, c_{k+2}, \ldots, c_{n+1}\right) .
$$

In any other case $g_{n+1, k}\left(c_{1}, \ldots, c_{n+1}\right)=0$. 
Clearly $\boldsymbol{F}_{n, 0}\left(c_{1}, c_{2}, \ldots, c_{n}\right)=\boldsymbol{G}_{n, 0}\left(c_{1}, c_{2}, \ldots, c_{n}\right)$ and $\boldsymbol{F}_{n, n}\left(c_{1}, c_{2}, \ldots, c_{n}\right)=\boldsymbol{G}_{n, n}\left(c_{1}, c_{2}, \ldots, c_{n}\right)$ for all $n=1,2, \ldots$. If $1 \leq k \leq n$, then by the induction hypothesis and by (19) and (20) we obtain that

$$
\boldsymbol{F}_{n+1, k}\left(c_{1}, c_{2}, \ldots, c_{n+1}\right) \equiv \boldsymbol{G}_{n+1, k}\left(c_{1}, c_{2}, \ldots, c_{n+1}\right)
$$

for $k=1,2, \ldots, n$. For if we collect the elements $f_{n+1, k}\left(c_{1}, c_{2}, \ldots, c_{n+1}\right)$ given by (19) and the elements $g_{n+1, k}\left(c_{1}, c_{2}, \ldots, c_{n+1}\right)$ given by $(20)$ for all $\left(c_{1}, c_{2}, \ldots, c_{n+1}\right) \in C_{n+1}$, and if we take into consideration that in both (19) and (20) one of the two terms on the right-hand side is necessarily 0 (possibly both terms are 0 ), then by the induction hypothesis we obtain (29). This completes the proof of the theorem.

Since

$$
\boldsymbol{F}_{n, k}\left(c_{1}, c_{2}, \ldots, c_{n}\right) \equiv \boldsymbol{G}_{n, k}\left(c_{1}, c_{2}, \ldots, c_{n}\right)
$$

is true for any $n=1,2, \ldots$ and $k=0,1, \ldots, n$ and for any choice of the real numbers $c_{1}, c_{2}, \ldots, c_{n}$, Theorem 1 can also be extended to interchangeable random variables.

\section{Stochastic Sequences}

Let us suppose that $\xi_{1}, \xi_{2}, \ldots, \xi_{n}$ are real random variables and write $\zeta_{r}=\xi_{1}+\xi_{2}+\ldots+\xi_{r}$ for $r=1,2, \ldots, n$ and $\zeta_{0}=0$. Define

$$
\omega_{n}(a)=\text { the number of subscripts } r=0,1, \ldots, n \text { for which } \zeta_{r} \leq a
$$

for $n=1,2, \ldots$ and $a \in(-\infty, \infty)$. Furthermore, define

$$
\eta_{n, j}=\inf \left\{a: \omega_{n}(a)>j\right\}
$$

for $j=0,1, \ldots, n$. Then

$$
\mathbf{P}\left\{\omega_{n}(a) \leq j\right\}=\mathbf{P}\left\{\eta_{n, j}>a\right\}
$$

for $j=0,1, \ldots, n$ and any real $a \in(-\infty, \infty)$. We observe that the variables $\eta_{n, j}(0 \leq j \leq n)$ are simply the variables $\zeta_{r}(0 \leq r \leq n)$ arranged in nondecreasing order of magnitude, that is,

In particular,

$$
\eta_{n, 0} \leq \eta_{n, 1} \leq \ldots \leq \eta_{n, n}
$$

and

$$
\eta_{n, n}=\max \left(\zeta_{0}, \zeta_{1}, \ldots, \zeta_{n}\right)
$$

$$
\eta_{n, 0}=\min \left(\zeta_{0}, \zeta_{1}, \ldots, \zeta_{n}\right)=-\max \left(-\zeta_{0},-\zeta_{1}, \ldots,-\zeta_{n}\right)
$$

Theorem 2: If $\xi_{1}, \xi_{2}, \ldots, \xi_{n}$ are interchangeable real random variables, we have

$$
\mathbf{P}\left\{\omega_{n}(a) \leq j\right\}=\mathbf{P}\left\{\eta_{n, j}>a\right\}=\mathbf{P}\left\{\max _{0 \leq r \leq j} \zeta_{r}+\min _{j \leq r \leq n}\left(\zeta_{r}-\zeta_{j}\right)>a\right\}
$$

for $0 \leq j \leq n$ and $a \in(-\infty, \infty)$.

Proof: For all $n$ ! permutations of any realization $\left(c_{1}, c_{2}, \ldots, c_{n}\right)$ of the random variables $\left(\xi_{1}, \xi_{2}, \ldots, \xi_{n}\right)$ we have identity $(30)$. This implies that for $0 \leq j \leq n$

$$
\eta_{n, j} \sim \max _{0 \leq r \leq j} \zeta_{r}+\min _{j \leq r \leq n}\left(\zeta_{r}-\zeta_{j}\right)
$$

where the symbol $\sim$ means that the random variables on both sides of (38) have the same distribution.

Note 1: By using a combinatorial method, in 1961, A. Brandt [4] already determined the distribution of $\omega_{n}(a)$ for interchangeable real random variables. Actually, he considered the random variable $N_{n}(a)$ defined as the number of subscripts $r=1,2, \ldots, n$ for which $\zeta_{r}>a$. In our notation $N_{n}(a)=n+1-\omega_{n}(a)$ if $a \geq 0$ and $N_{n}(a)=n-\omega_{n}(a)$ if $a<0$. By the result of A. Brandt 
[4],

$$
\begin{gathered}
\mathbf{P}\left\{N_{n}(a)=k\right\}=\mathbf{P}\left\{\max _{k<i \leq n}\left(\zeta_{i}-\zeta_{k}\right)+\min _{1 \leq i \leq k} \zeta_{i} \leq a \text { and } \min _{1 \leq i \leq k} \zeta_{i}>a\right\} \\
+\mathbf{P}\left\{\max _{k<i \leq n}\left(\zeta_{i}-\zeta_{k}\right)+\min _{1 \leq i \leq k} \zeta_{i}>a \text { and } \max _{k<i \leq n}\left(\zeta_{i}-\zeta_{k}\right) \leq a\right\}
\end{gathered}
$$

for $0<k<n$ and any $a \in(-\infty, \infty)$.

Note 2: If $\xi_{1}, \xi_{2}, \ldots, \xi_{n}$ are interchangeable random variables having finite expectations, then by a theorem of M. Kac [12]

for $1 \leq j \leq n$. See also L. Takács [22]. Thus by (38),

$$
\mathbf{E}\left\{\max \left(\zeta_{0}, \zeta_{1}, \ldots, \zeta_{j}\right)\right\}=\sum_{i=1}^{j} \frac{1}{i} \mathbf{E}\left\{\zeta_{i}^{+}\right\}
$$

for $0 \leq j \leq n$.

$$
\mathbf{E}\left\{\eta_{n, j}\right\}=\sum_{1 \leq i \leq j} \frac{1}{i} \mathbf{E}\left\{\zeta_{i}^{+}\right\}+\sum_{1 \leq i \leq n-j} \frac{1}{i} \mathbf{E}\left\{\zeta_{i}^{-}\right\}
$$

If, in particular, $\xi_{1}, \xi_{2}, \ldots, \xi_{n}$ are independent and identically distributed random variables, then Theorem 2 is applicable and in this case the two random variables on the right-hand side of (38) are independent. Thus we can write that

$$
\eta_{n, j}=\eta_{j, j}+\bar{\eta}_{n-j, 0}
$$

for $0 \leq j \leq n$ where $\eta_{j, j}$ and $\bar{\eta}_{n-j, 0}$ are independent and $\bar{\eta}_{n-j, 0}$ has the same distribution as $\eta_{n-j, 0}$

Note 3: In the case of independent and identically distributed random variables $\xi_{1}, \xi_{2}, \ldots, \xi_{n}$, relation (42) can also be deduced from a result of F. Pollaczek [15] found in 1952. Let us define

$$
\Gamma_{n, j}(s)=\mathbf{E}\left\{e^{-s \eta} n, j\right\}
$$

for $0 \leq j \leq n$ and $\Re(s)=0$. By Pollaczek's result

$$
\sum_{n=0}^{\infty} \sum_{j=0}^{n} \Gamma_{n, j}(s) \rho^{n} w^{j}=\left(\sum_{n=0}^{\infty} \Gamma_{n, 0}(s) \rho^{n}\right)\left(\sum_{n=0}^{\infty} \Gamma_{n, n}(s)(\rho w)^{n}\right)
$$

for $\mathscr{R}(s)=0,|\rho|<1$ and $|\rho w|<1$. If we form the coefficient of $\rho^{n} w^{j}$ on both sides of (44) we obtain that

$$
\Gamma_{n, j}(s)=\Gamma_{j, j}(s) \Gamma_{n-j, 0}(s)
$$

for $0 \leq j \leq n$ and $\mathscr{R}(s)=0$. This implies (42).

Example 1: Let us suppose that $\left\{\xi_{r}, r \geq 1\right\}$ is a sequence of independent and identically distributed random variables for which

$$
\mathbf{P}\left\{\xi_{r}=1\right\}=p \text { and } \mathbf{P}\left\{\xi_{r}=-1\right\}=q
$$

where $p>0, q>0$ and $p+q=1$. Then $\left\{\zeta_{r}, r \geq 0\right\}$ describes a random walk on the real line and

$$
\mathbf{P}\left\{\zeta_{n}=2 j-n\right\}=\left(\begin{array}{c}
n \\
j
\end{array}\right) p^{j} q^{n-j}
$$

for $0 \leq j \leq n$. By the reflection principle we obtain that

and

$$
\mathbf{P}\left\{\eta_{n, n}<k\right\}=\mathbf{P}\left\{\zeta_{n}<k\right\}-\left(\frac{p}{q}\right)^{k} \mathbf{P}\left\{\zeta_{n}<-k\right\}
$$

$$
\mathbf{P}\left\{-\eta_{n, 0}<k\right\}=\mathbf{P}\left\{\zeta_{n}>-k\right\}-\left(\frac{q}{p}\right)^{k} \mathbf{P}\left\{\zeta_{n}>k\right\}
$$

for $k>0$ and $n \geq 1$. See L. Takács [21]. Probabilities (48) and (49) completely determine the distribution for $\omega_{n}(a)$ for $n \geq 1$ and $a \in(-\infty, \infty)$. 
In this particular case, the distribution of $\omega_{n}(a)$ can also be expressed in a simpler form. Let us define $\rho(k)$ as the first passage time through $k$, that is,

$$
\rho(k)=\inf \left\{r: \zeta_{r}=k \text { and } r \geq 0\right\}
$$

for $k=0, \pm 1, \pm 2, \ldots$. If $1 \leq k \leq n$, then

$$
\mathbf{P}\{\rho(k) \leq n\}=\mathbf{P}\left\{\zeta_{n} \geq k\right\}+\left(\frac{p}{q}\right)^{k} \mathbf{p}\left\{\zeta_{n}<-k\right\} .
$$

By symmetry

for $j \geq 0$.

$$
\mathbf{P}\{\rho(-k)=j\}=\left(\frac{q}{p}\right)^{k} \mathbf{P}\{\rho(k)=j\}
$$

If $n \geq 1$, then

$$
\mathbf{P}\left\{\omega_{n}(k)=j\right\}=[\mathbf{P}\{\rho(k+1) \geq j\}-\mathbf{P}\{\rho(k) \geq j\}][p-q \mathbf{P}\{\rho(1) \leq n-j\}]
$$

for $1 \leq k+1 \leq j \leq n$ and

$$
\mathbf{P}\left\{\omega_{n}(k)=n+1\right\}=\mathbf{P}\{\rho(1)>n\}
$$

for $0 \leq k \leq n$. See L. Takács [23].

\section{Stochastic Processes}

Let $\{\chi(u), u \geq 0\}$ be a separable homogeneous stochastic process with independent increments for which $\mathbf{P}\{\chi(0)=0\}=1$. Define

$$
\tau(\alpha)=\int_{0}^{1} \delta(\chi(u) \leq \alpha) d u
$$

for $\alpha \in(-\infty, \infty)$, that is, $\tau(\alpha)$ is the sojourn time of the process $\{\chi(u), u \geq 0\}$ spent in the set $(-\infty, \alpha]$ in the time interval $(0,1)$. We also define

$$
\gamma(x)=\inf \{\alpha: \tau(\alpha)>x\}
$$

for $0<x<1$, that is, $\{\gamma(x), 0<x<1\}$ is the inverse process of $\{\tau(\alpha),-\infty<\alpha<\infty\}$. Obviously,

$$
\mathbf{P}\{\tau(\alpha) \leq x\}=\mathrm{P}\{\gamma(x)>\alpha\}
$$

for $0<x<1$ and $-\infty<\alpha<\infty$.

Theorem 3: We have

for $0<x<1$ and $\alpha \in(-\infty, \infty)$.

$$
\mathbf{P}\{\tau(\alpha) \leq x\}=\mathbf{P}\{\gamma(x)>\alpha\}=\mathbf{P}\left\{\sup _{0 \leq u \leq x} \chi(u)+\inf _{x \leq u \leq 1}[\chi(u)-\chi(x)]>\alpha\right\}
$$

Proof: Let us assume that in Theorem 2

$$
\zeta_{r}=\chi(r / n)
$$

for $r=0,1, \ldots, n$. If $n \rightarrow \infty$, then the process $\left\{\zeta_{[n u]}, 0 \leq u \leq 1\right\}$ converges weakly to the process $\{\chi(u), 0 \leq u \leq 1\}$. Since $\tau(\alpha)$ is a continuous functional of the process $\{\chi(u), 0 \leq u \leq 1\}$, we can conclude that if $a=\alpha$ and $j=[n x]$ where $0<x<1$, then

$$
\lim _{n \rightarrow \infty} \mathbf{P}\left\{\omega_{n}(a) \leq j\right\}=\mathbf{P}\{\tau(\alpha \leq x\}
$$

for $0<x<1$ provided that $x$ is a continuity point of $\mathbf{P}\{\tau(\alpha) \leq x\}$. By (33), (57) and (60) we obtain that if $a=\alpha$ and $j=[n x]$ where $0<x<1$, then 


$$
\lim _{n \rightarrow \infty} \mathbf{P}\left\{\eta_{n, j}>a\right\}=\mathbf{P}\{\gamma(x)>\alpha\}
$$

is also true. This completes the proof of Theorem 3.

Accordingly, if we know the distribution functions

and

$$
\mathbf{P}\left\{\sup _{0 \leq u \leq t} \chi(u) \leq \alpha\right\}=G(\alpha, t)
$$

$$
\mathbf{P}\left\{\sup _{0 \leq u \leq t}[-\chi(u)] \leq \alpha\right\}=H(\alpha, t)
$$

for $0 \leq t \leq 1$, then, by Theorem 3 , we obtain that

$$
\mathbf{P}\{\tau(\alpha) \leq x\}=\mathbf{P}\{\gamma(x)>\alpha\}=\int_{0}^{\infty}[1-G(\alpha+u, x)] d_{u} H(u, 1-x)
$$

for $0<x<1$ and $\alpha \in(-\infty, \infty)$.

In 1957, G. Baxter and M.D. Donsker [3] gave a general method for the determination of (62) and (63). In 1984, D.V. Gusak [10] studied the problem of finding the distribution of $\tau(\alpha)$; however, it does not seem that Theorem 3 can be deduced from his results.

Note 4: If we assume that $\{\chi(u), 0 \leq u \leq 1\}$ is a stochastic process with interchangeable increments, then Theorem 3 is still valid.

Example 2: Let $\{\xi(u), u \geq 0\}$ be a standard Brownian motion process. We have $\mathbf{P}\{\xi(u) \leq$ $x\}=\Phi(x / \sqrt{u})$ for $u>0$ where $\Phi(x)$ is defined by (1). Let us consider the process $\{\xi(u)+m x u$, $u \geq 0\}$ where $m$ is a real number. Define

$$
\tau(\alpha, m)=\int_{0}^{1} \delta(\xi(u)+m u \leq \alpha) d u
$$

that is, $\tau(\alpha, m)$ is the sojourn time of the process $\{\xi(u)+m u, u \geq 0\}$ spent in the set $(-\infty, \alpha]$ in the time interval $(0,1)$. We also define

$$
\gamma(x, m)=\inf \{\alpha: \tau(\alpha, m)>x\}
$$

for $0<x<1$, that is, $\{\gamma(x, m), 0<x<1\}$ is the inverse process of $\{\tau(\alpha, m),-\infty<\alpha<\infty\}$. We have

$$
\mathbf{P}\{\tau(\alpha, m) \leq x\}=\mathbf{P}\{\gamma(x, m)>\alpha\}
$$

for $0<x<1$ and $\alpha \in(-\infty, \infty)$. To find the distribution of $\tau(\alpha, m)$ or $\gamma(x, m)$ by Theorem 3 it is sufficient to determine the following probability

$$
\mathbf{P}\left\{\sup _{0 \leq u \leq t}[\xi(u)+m u] \leq \alpha\right\}=F(\alpha, m, t)
$$

for $0<t<1$ and $m \in(-\infty, \infty)$. Obviously, $F(\alpha, m, t)=F(\alpha / \sqrt{t}, m \sqrt{t}, 1)$ for $t>0$. If we consider Example 1 and assume that in the random walk $\left\{\zeta_{r}, r \geq 0\right\}$

$$
p=p_{n}=\frac{1}{2}+\frac{m}{2 \sqrt{n}} \text { and } q=q_{n}=\frac{1}{2}-\frac{m}{2 \sqrt{n}}
$$

for $n>m^{2}$, then the process $\left\{\zeta_{[n u]} / \sqrt{n}, 0 \leq u \leq 1\right\}$ converges weakly to the process $\{\xi(u)+m u$, $0 \leq u \leq 1\}$ as $n \rightarrow \infty$. Since the supremum is a continuous functional of the process $\{\xi(u)+m u$, $0 \leq u \leq 1\}$, we can conclude that if $k=[\alpha \sqrt{n}]$ where $\alpha>0$, then

$$
\lim _{n \rightarrow \infty} \mathbf{P}\left\{\max \left(\zeta_{0}, \zeta_{1}, \ldots, \zeta_{n}\right)<k\right\}=F(\alpha, m, 1) .
$$

If we apply the central limit theorem to the random variables $\zeta_{n}=\xi_{1}+\xi_{2}+\ldots+\xi_{n}$ as $n \rightarrow \infty$, then by (48) we obtain $F(\alpha, m, 1)$. Thus 


$$
F(\alpha, m, t)=\Phi\left(\frac{\alpha-m t}{\sqrt{t}}\right)-e^{2 m \alpha} \Phi\left(\frac{-\alpha-m t}{\sqrt{t}}\right)
$$

and

$$
f(\alpha, m, t)=\frac{\partial F(\alpha, m, t)}{\partial \alpha}=2\left[\varphi\left(\frac{\alpha-m t}{\sqrt{t}}\right) \frac{1}{\sqrt{t}}-m e^{2 m \alpha} \Phi\left(\frac{-\alpha-m t}{\sqrt{t}}\right)\right]
$$

for $t>0, \alpha>0$, and $m \in(-\infty, \infty)$, where

$$
\varphi(x)=\frac{1}{\sqrt{2 \pi}} e^{-x^{2} / 2}
$$

is the normal density function. If $\alpha \leq 0$, then $F(\alpha, m, t)=0$.

Now we have $G(\alpha, t)=F(\alpha, m, t)$ and $H(\alpha, t)=F(\alpha,-m, t)$, and by $(58)$

$$
\mathbf{P}\{\tau(\alpha, m) \leq x\}=\int_{0}^{\infty}[1-F(\alpha+u, m, x)] f(u,-m, 1-x) d u
$$

for $0<x<1$ and $\alpha \in(-\infty, \infty)$.

Several recent papers are concerned with the problem of finding the distribution function of $\tau(\alpha, m)$. By the results of J. Akahori [1] and A. Dassios [6] the distribution function of $\tau(\alpha, m)$ can be expressed in the form of a double integral. The above formula (74) is in agreement with their result. Both authors applied the method of M. Kac [11] in their papers. In finding the density function of $\gamma(x, m)$, Dassios observed that this density function is the convolution of the density functions of two random variables. One of the variables is $\sup _{0}<u<x \chi(u)$ and the other has the same distribution as $\inf _{0 \leq u \leq 1-x} \chi(u)$ where $\chi(u)=\xi(u)+m u$ for $u \geq 0$. Thus Dassios concluded that Theorem 3 is true for the Brownian motion with drive. As we have seen, Theorem 3 is true more generally for homogeneous stochastic processes with independent increments. Recently P. Embrechts, L.C.G. Rogers and M. Yor [9] gave two different proofs for Dassio's result.

By using formula (53) we can derive that

$$
\mathbf{P}\{\tau(\alpha, m) \leq x\}=\frac{1}{2} \int_{0}^{x} f(\alpha, m, u) f(0,-m, 1-u) d u
$$

for $0<x<1$ and

$$
\mathbf{P}\{\tau(\alpha, m)=1\}=\Phi(\alpha-m)-e^{2 m \alpha} \Phi(-\alpha-m)
$$

for $\alpha \geq 0$ and $m \in(-\infty, \infty)$ where $\Phi(x)$ is defined by (1). See L. Takács [23].

\section{Exact Distributions}

Let us consider again a stochastic process $\{\zeta(u), u \geq 0\}$ with state space $A \cup B$ where $A$ and $B$ are disjoint sets. Let us assume now that in any finite interval $(0, t)$ the process changes states only a finite number of times with probability one. Let us suppose that $\mathbf{P}\{\zeta(0) \in A\}=1$ and denote by $\alpha_{1}, \beta_{1}, \alpha_{2}, \beta_{2}, \ldots$ the lengths of the successive intervals spent in states $A$ and $B$ respectively in the interval $(0, \infty)$. Denote by $\alpha(t)$ the total time spent in state $A$ in the time interval $(0, t)$ and by $\beta(t)$ the total time spent in state $B$ in the time interval $(0, t)$. Obviously, $\alpha(t)$ and $\beta(t)$ are random variables and $\alpha(t)+\beta(t)=t$ for all $t \geq 0$. Our aim is to determine the distributions of $\alpha(t)$ and $\beta(t)$ for $t \geq 0$ and their asymptotic distributions as $t \rightarrow \infty$.

Define

$$
\gamma_{n}=\alpha_{1}+\alpha_{2}+\ldots+\alpha_{n}
$$

for $n \geq 1$ and $\gamma_{0}=0 ;$ furthermore,

$$
\delta_{n}=\beta_{1}+\beta_{2}+\ldots+\beta_{n}
$$


for $n \geq 1$ and $\delta_{0}=0$.

Theorem 4: If $0 \leq x<t$, then

$$
\mathbf{P}\{\beta(t) \leq x\}=\sum_{n=0}^{\infty}\left[\mathbf{P}\left\{\delta_{n} \leq x, \gamma_{n}<t-x\right\}-\mathbf{P}\left\{\delta_{n} \leq x, \gamma_{n+1}<t-x\right\}\right]
$$

and if $0<x \leq t$, then

$$
\mathbf{P}\{\alpha(t)<x\}=\sum_{n=1}^{\infty}\left[\mathbf{P}\left\{\gamma_{n}<x, \delta_{n-1} \leq t-x\right\}-\mathbf{P}\left\{\gamma_{n}<x, \delta_{n} \leq t-x\right\}\right] .
$$

Proof: Since $\mathbf{P}\{\alpha(t)<x\}=1-\mathbf{P}\{\beta(t) \leq t-x\}$ for $0 \leq x \leq t$, it is sufficient to prove (79). For $0 \leq x<t$ denote by $\tau=\tau(t-x)$ the smallest $u \in[0, \infty)$ for which $\alpha(u)=t-x$ if such a $u$ exists. (If such a $u$ does not exist, then (79) is trivially true.) Then $\zeta(\tau) \in A$ and we have

$$
\{\beta(t) \leq x\} \equiv\{\beta(\tau) \leq x\}
$$

This follows from the identities

$$
\{\beta(t) \leq x\} \equiv\{\alpha(\tau) \leq \alpha(t)\} \equiv\{\tau \leq t\} \equiv\{\alpha(\tau)+\beta(\tau) \leq t\} \equiv\{\beta(\tau) \leq x\} .
$$

Here we used that $\alpha(t)+\beta(t)=t$ for all $t \geq 0$, and that $\alpha(t)$ and $\beta(t)$ are nondecreasing continuous functions of $t$ for $0 \leq t<\infty$.

Since $\beta(\tau)=\delta_{n}(n=0,1,2, \ldots)$ if $\gamma_{n}<t-x \leq \gamma_{n+1}$, it follows from (82) that

$$
\mathbf{P}\{\beta(t) \leq x\}=\sum_{n=0}^{\infty} \mathbf{P}\left\{\delta_{n} \leq x \text { and } \gamma_{n}<t-x \leq \gamma_{n+1}\right\}
$$

for $0 \leq x<t$ which proves $(79)$.

If for each $t \geq 0$ we define $\rho(t)$ as a discrete random variable which takes on only nonnegative integers and satisfies the relation

$$
\{\rho(t)<n\} \equiv\left\{\gamma_{n} \geq t\right\}
$$

for all $t \geq 0$ and $n=1,2, \ldots$, then we can write

$$
\mathbf{P}\{\beta(t) \leq x\}=\mathbf{P}\left\{\delta_{\rho(t-x)} \leq x\right\}
$$

for $0 \leq x \leq t$. We note that $\mathbf{P}\{\rho(0)=0\}=1$.

Now $\delta_{\rho(t)}$ is the sum of a random number of random variables. If we can determine the distribution of $\delta_{\rho(t)}$ for all $t \geq 0$, then by (85) we can also determine the distribution of $\beta(t)$ for all $t \geq 0$. If the two sequences $\left\{\alpha_{n}\right\}$ and $\left\{\beta_{n}\right\}$ are independent, then $\left\{\delta_{n}\right\}$ and $\{\rho(t), t \geq 0\}$ are also independent, and the problem of finding the distribution of $\beta(t)$ can be reduced to the problem of finding the distribution of the sum of a random number of random variables where the number of variables and the variables themselves are independent.

In what follows, we assume that the two sequences $\left\{\alpha_{n}, n \geq 0\right\}$ and $\left\{\beta_{n}, n \geq 0\right\}$ are independent. If in addition, the random variables $\left\{\alpha_{n}, n \geq 0\right\}$ are identically distributed independent random variables and the random variables $\left\{\beta_{n}, n \geq 0\right\}$ are also identically distributed independent random variables, then as an alternative we can determine the distribution of $\beta(t)$ by using Laplace transforms. In this case, let us define

and

$$
\mathbf{E}\left\{e^{-s \alpha} n\right\}=\varphi(s)
$$

$$
\mathbf{E}\left\{e^{-s \beta} n=\psi(s)\right.
$$

for $\mathscr{R}(s) \geq 0$. Then by (84), 


$$
q \int_{0}^{\infty} e^{-q t} \mathbf{E}\left\{e^{-s \delta} \rho(t)\right\} d t=\frac{1-\varphi(q)}{1-\varphi(q) \psi(s)}
$$

for $\mathscr{R}(s) \geq 0$ and $\Re_{(}(q)>0$. If we know $\varphi(s)$ and $\psi(s)$, then by inverting (88) we can determine the distribution of $\delta_{\rho(t)}$, and $\mathbf{P}\{\beta(t) \leq x\}$ can be obtained by (85).

Finally, we would like to mention that if $P_{A}(t)=\mathbf{P}\{\zeta(t) \in A\}$, then we have

$$
\int_{0}^{\infty} e^{-s t} P_{A}(t) d t=\frac{1-\varphi(s)}{s[1-\varphi(s) \psi(s)]}
$$

for $\mathscr{R}(s)>0$. If $P_{A}(t)$ and $\varphi(s)$ are known, then by (89) we can determine $\psi(s)$. See the following example.

Example 3: Let $\{\zeta(t), t \geq 0\}$ be a homogeneous irreducible Markov process with finite state space $I$ and transition probability matrix $\mathbf{P}(t)=\left[p_{i, k}(t)\right]_{i, k \in I^{*}}$ Let $A=\{i\}$ and $B=I \backslash\{i\}$ where $i \in I$ is a given state. Let us suppose that $\mathbf{P}\{\zeta(0)=i\}=1$. In this case, $P_{A}(t)=p_{i, i}(t)$ and

$$
\mathbf{P}\left\{\alpha_{n} \leq x\right\}=1-e^{-\lambda_{i} x}
$$

if $x \geq 0$ where $\lambda_{i}=-p_{i, i}^{\prime}(0)$. Hence $\varphi(s)=\lambda_{i} /\left(\lambda_{i}+s\right)$. By (89) we obtain $\psi(s)$. Thus we have all the ingredients for the determination of the distribution of $\beta(t)$.

For the sojourn times of two-state Markov chains various asymptotic distributions were obtained by R.L. Dobrushin [7].

\section{Limit Distributions}

Let us assume that the two sequences $\left\{\alpha_{n}, n \geq 0\right\}$ and $\left\{\beta_{n}, n \geq 0\right\}$ are independent. If we know the asymptotic distributions of $\gamma_{n}=\alpha_{1}+\alpha_{2}+\ldots+\alpha_{n}$ and $\delta_{n}=\beta_{1}+\beta_{2}+\ldots+\beta_{n}$ as $n \rightarrow \infty$, then we expect that the asymptotic distribution of $\beta(t)$ for $t \rightarrow \infty$ is determined by these two distributions. This is indeed the case. For a detailed discussion, see L. Takács [18, 19]. Here we consider only a particular case.

Let us assume that both $\gamma_{n}$ and $\delta_{n}$ have an asymptotic normal distribution if $n \rightarrow \infty$, namely,

and

$$
\lim _{n \rightarrow \infty} \mathbf{P}\left\{\frac{\gamma_{n}-n a}{\sigma_{a} \sqrt{n}} \leq x\right\}=\Phi(x)
$$

$$
\lim _{n \rightarrow \infty} \mathbf{P}\left\{\frac{\delta_{n}-n b}{\sigma_{b} \sqrt{n}} \leq x\right\}=\Phi(x)
$$

where $\Phi(x)$ is the normal distribution function defined by (1) and $a, b, \sigma_{a}$ and $\sigma_{b}$ are positive real constants. We can simply write that $\gamma_{n} \sim N\left(n a, n \sigma_{a}^{2}\right)$ as $n \rightarrow \infty$ and $\delta_{n} \sim N\left(n b, n \sigma_{b}^{2}\right)$ as $n \rightarrow \infty$. By (84) we can prove that

$$
\rho(t) \sim N\left(t / a, \sigma_{a}^{2} t / a^{3}\right)
$$

as $t \rightarrow \infty$. Now $\delta_{\rho(t)}$ can be interpreted as a sum of a random number of random variables. By working with characteristic functions, H. Robbins $[16,17]$ determined the asymptotic distributions of such sums. By his results, we can conclude that

$$
\delta_{\rho(t)} \sim N\left(b t / a,\left(a^{2} \sigma_{b}^{2}+b^{2} \sigma_{a}^{2}\right) t / a^{3}\right)
$$

as $t \rightarrow \infty$. This result can be proved in a simple way by a result of R.L. Dobrushin [8] for compound random functions. The substance of Dobrushin's idea is that the asymptotic distribution of $\delta_{\rho(t)}$ is independent of the particular choices of $\left\{\delta_{n}\right\}$ and $\{\rho(t)\}$; it depends only on their asymp 
totic distributions. Consequently, we may replace $\delta_{n}$ by

and $\rho(t)$ by

$$
\delta_{n}^{*}=n b+\sqrt{n} \sigma_{b} \delta
$$

$$
\rho^{*}(t)=t / a+t \sigma_{b} \rho / a^{3 / 2}
$$

where $\delta$ and $\rho$ are independent random variables having the same normal distribution defined by (1). Since $\delta_{\rho(t)}$ has the same asymptotic distribution as $\delta_{\rho^{*}(t)}^{*}$ if $t \rightarrow \infty$, we can conclude that

$$
\lim _{t \rightarrow \infty} \mathbf{P}\left\{\frac{\delta_{\rho(t)}-(b / a) t}{\sqrt{t}} \leq x\right\}=\mathbf{P}\left\{\frac{b \sigma_{a} \rho+a \sigma_{\delta}}{a^{3 / 2}} \leq x\right\} .
$$

This proves (94). Finally, by (85) and (94) we obtain that

$$
\beta(t) \sim N\left(b t /(a+b),\left(a^{2} \sigma_{b}^{2}+b^{2} \sigma_{a}^{2}\right) t /(a+b)^{3}\right)
$$

as $t \rightarrow \infty$. For the asymptotic distribution of $\beta(t)$, many more examples can be found in $\mathrm{L}$. Takács [18, 19].

By using a limit theorem of F.J. Anscombe [2], we can find the asymptotic distribution of $\beta(t)$ as $t \rightarrow \infty$ for stochastic processes in which $\left(\alpha_{n}, \beta_{n}\right)$ are independent vector random variables. For details, see L. Takács $[19,20]$.

Example 4: Let us suppose that in the time interval $(0, \infty)$ customers arrive at a counter in accordance with a Poisson process of intensity $\lambda$ and are served by one server. The server is always busy if there is at least one customer at the counter. The service times are assumed to be independent identically distributed random variables having a finite expectation $\alpha$ and a finite variance $\sigma^{2}$ and independent of the arrival times. It is also assumed that $\lambda \alpha<1$. Denote by $\beta(t)$, the total occupation time of the server in the time interval $(0, t)$. Now the lengths of the successive idle periods, $\alpha_{n}(n=1,2, \ldots)$ and the lengths of the successive busy periods, $\beta_{n}(n=1,2, \ldots)$ are independent sequences of independent and identically distributed random variables and by $(98) \beta(t)$ has an asymptotic normal distribution. The parameters in (98) are: $a=1 / \lambda, \sigma_{a}^{2}=1 / \lambda^{2}, b=\alpha /(1-\lambda \alpha)$ and

$$
\sigma_{b}^{2}=\left(\sigma^{2}+\lambda \alpha^{3}\right) /(1-\lambda \alpha)^{3} .
$$

Thus,

$$
\lim _{t \rightarrow \infty} \mathbf{P}\left\{\frac{\beta(t)-\lambda \alpha t}{\sqrt{\lambda\left(\sigma^{2}+\alpha^{2}\right) t}} \leq x\right\}=\Phi(x)
$$

where $\Phi(x)$ is defined by (1). For further details and extensions, see L. Takács [19].

\section{References}

[1] Akahori, J., Some formulae for a new type of path-dependent option, Ann. Appl. Prob. 5 (1995), 383-388.

[2] Anscombe, F.J., Large-sample theory of sequential estimation, Proc. Cambridge Phil. Soc. 48 (1952), 600-607.

[3] Baxter, G. and Donsker, M.D., On the distribution of the supremum functional for processes with stationary independent increments, Trans. Amer. Math. Soc. 85 (1957), 73-87.

[4] Brandt, A., A generalization of a combinatorial theorem of Sparre Andersen about sums of random variables, Math. Scand. 9 (1961), 352-358.

[5] Darling, D.A. and Kac, M., On occupation times for Markov processes, Trans. Amer. Math. Soc. 84 (1957), 444-458.

[6] Dassios, A., The distribution of the quantile of a Brownian motion with drift and the 
pricing of related path-dependent options, Ann. Appl. Prob. 5 (1995), 389-398.

[7] Dobrushin, R.L., Limit theorems for a Markov chain of two states, Izv. Akad. Nauk SSSR. Ser. Math. 17 (1953), 291-330 (Russian). [English trans.: Selected Trans. in Math. Stat. and Prob. 1, AMS and IMS (1961), 97-134.]

[8] Dobrushin, R.L., Lemma on the limit of compound random functions, Uspehi Mat. Nauk 10:2 (1955), 157-159 (Russian).

[9] Embrechts, P., Rogers, L.C.G., and Yor, M., A proof of Dassios' representation of the $\alpha$ quantile of Brownian motion with drift, Ann. Appl. Prob. 5 (1995), 757-767.

[10] Gusak, D.V., Distribution of the sojourn time of a homogeneous process with independent increments above an arbitrary level, Theor. Prob. Appl. 28 (1984), 503-514.

[11] Kac, M., On distributions of certain Wiener functionals, Trans. Amer. Math. Soc. 65 (1949), 1-13. [Reprinted in Mark Kac: Probability, Number Theory, and Statistical Physics. Selected Papers. Ed. by K. Baclawski and M.D. Donsker, The MIT Press, Cambridge, MA (1979), 268-280.]

[12] Kac, M., Toeplitz matrices, translations kernels and a related problem in probability theory, Duke Math. J. 21 (1954), 501-509. [Reprinted in Mark Kac: Probability, Number Theory, and Statistical Physics. Selected Papers. Ed. by K. Baclawski and M.D. Donsker, The MIT Press, Cambridge, MA (1979), 379-387.]

[13] Lévy, P., Sur un problème de M. Marcinkiewicz, Comptes Rendus Acad. Sci. Paris 208 (1939), 318-321. [Errata: Ibid, p. 776.]

[14] Lévy, P., Sur certains processus stochastiques homogènes, Compositio Math. 7 (1939), 283339.

[15] Pollaczek, F., Fonctions caractéristiques de certaines répartitions définies au moyen de la notion d'ordre. Application à la théorie des attentes, C.R. Acad. Sci. Paris 234 (1952), 2334-2336.

[16] Robbins, H., The asymptotic distribution of the sum of a random number of random variables, Bull. Amer. Math. Soc. 54 (1948), 1151-1161.

[17] Robbins, H., On the asymptotic distribution of the sum of a random number of random variables, Proc. Nat. Acad. Sci. USA 34 (1948), 162-163.

[18] Takács, L., On a sojourn time problem in the theory of stochastic processes, Trans. Amer. Math. Soc. 93 (1959), 531-540.

[19] Takács, L., Occupation time problems in the theory of queues, Math. Methods in Queueing Theory, Ed. by A.B. Clarke. Lecture Notes in Economics and Mathematical Systems 98, Springer-Verlag, Berlin (1974), 91-131.

[20] Takács, L., Sojourn time problems, Ann. Prob. 2 (1974), 420-431.

[21] Takács, L., Fluctuation problems for Bernoulli trials, SIAM Review 21 (1979), 222-228.

[22] Takács, L., On a combinatorial theorem related to a theorem of G. Szegö, J. Comb. Theory Ser. A 30 (1981), 345-348.

[23] Takács, L., On a generalization of the arc-sine law, Ann. Appl. Prob. 6:3 (1996), 10351040.

[24] Yor, M., The distribution of Brownian quantiles, J. Appl. Prob. 32 (1995), 405-416. 


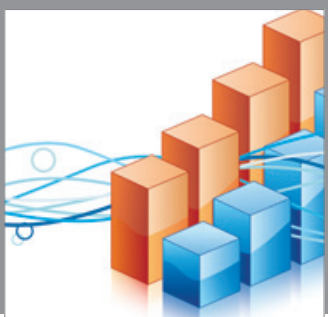

Advances in

Operations Research

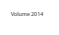

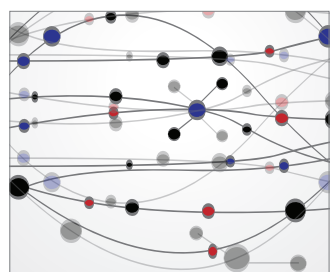

\section{The Scientific} World Journal
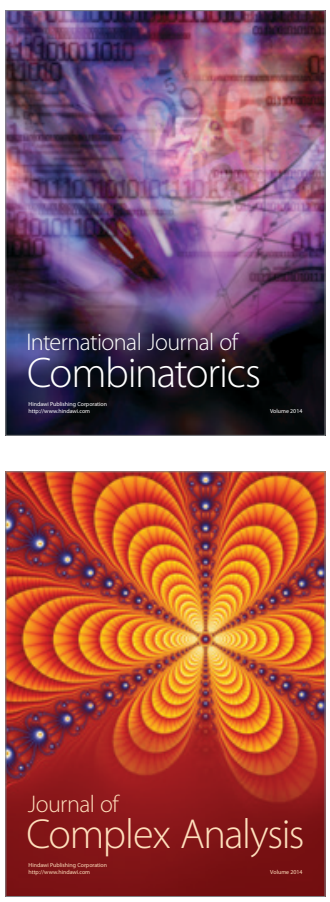

International Journal of

Mathematics and

Mathematical

Sciences
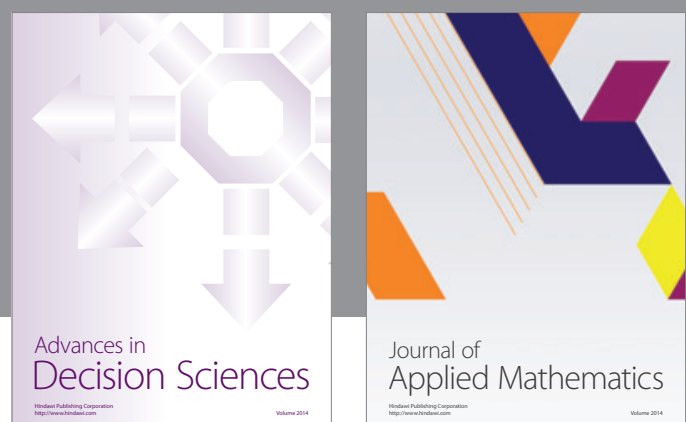

Journal of

Applied Mathematics
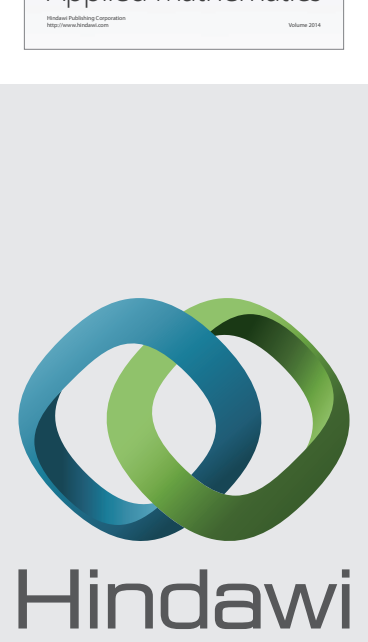

Submit your manuscripts at http://www.hindawi.com
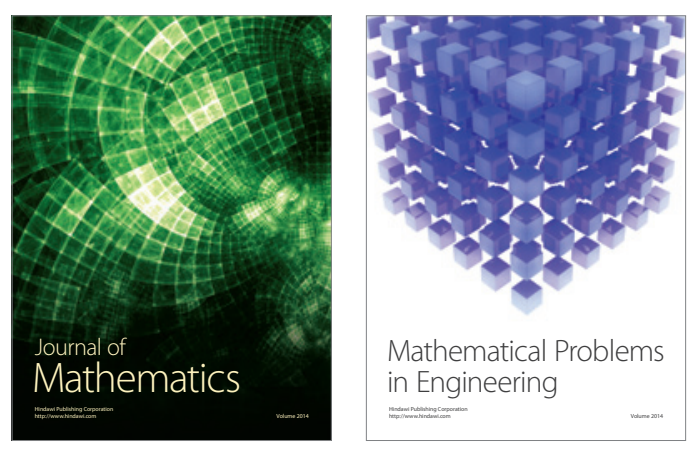

Mathematical Problems in Engineering
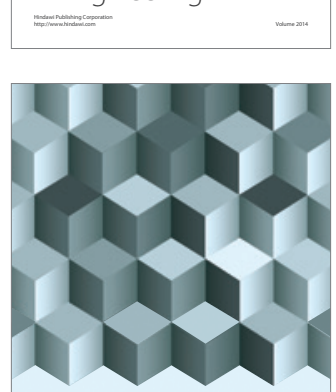

Journal of

Function Spaces
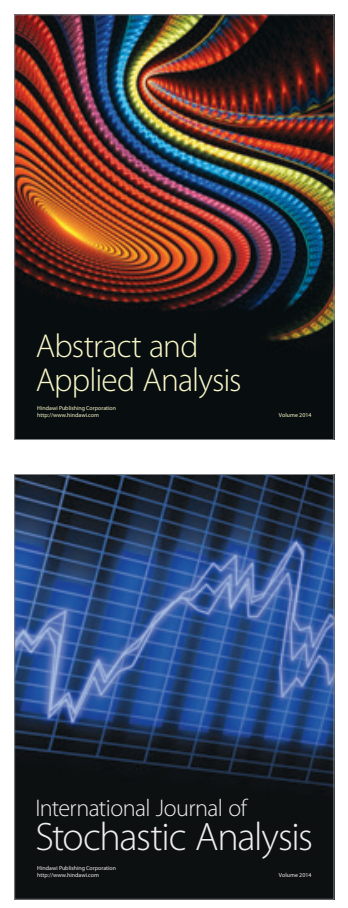

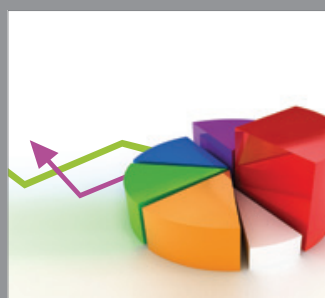

ournal of

Probability and Statistics

Promensencen
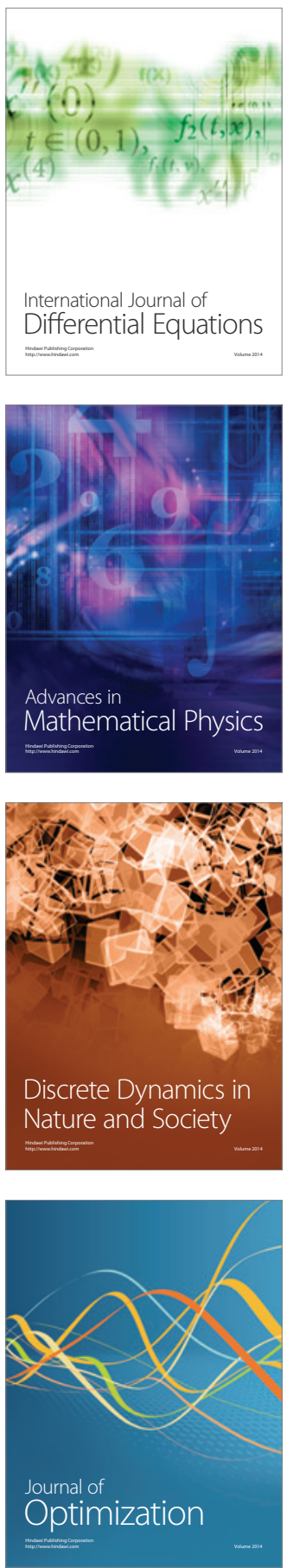